\title{
Stenasellus skopljensis thermalis ssp.n. (Crustacea, Isopoda) d'une source chaude en Bosnie
}

\author{
Milan Meštrov et Romana Lattinger-Penko ${ }^{1)}$
}

Avec planches 97 (1)-99 (3)

Nous avons fait, au cours des quelques dernières années, des recherches biologiques étendues sur les eaux souterraines accompagnant les affluents de droite de la Save, sur le territoire de Bosnie.

Cet article traite plus particuliérement de la trouvaille du genre Stenasellus dans la source chaude de Banja Luka, près de la rivière Vrbas. Le premier exemplaire () a été trouvé dans les alluvions sablonneuses (14.9. 1961.) le long de la rive droite du Vrbas, où affluent de faibles courants d'eau chaude des sources thermales voisines. Plus tard, l'examen de sources voisines nous a permis de recueillir un nombre plus important d'exemplaires uniquement dans la source chaude «Šugavac»: le 13.2.1966. (5 ot et 10 \%) et le 19.3. 1967. ( $8 \sigma^{\star}$ et 14 क ). Au total: 13 ot et 25 ㅇ).

La source est captée sous forme d'un petit bassin au fond duquel l'eau jaillit doucement à plusieurs endroits, (temp. $24^{\circ} \mathrm{C}$ ). Les animaux se tiennent dans une vase peu épaisse, sous de petites branches immergées et des feuilles pourries. Ces animaux, étant donné leurs particularités morphologiques et le milieu dans lequel ils vivent, peuvent être considérés comme une sous-espèce nouvelle et dénommés Stenasellus skopljensis thermalis. Nous n'avons jamais trouvé cette espèce au cours de recherches répétées dans les nappes phréatiques froides voisines de la source de Banja Luka.

Le genre Stenasellus (Magniez, 1966) comprend 17 espèces et sous-espèces eurasiennes, et 1 espèce de l'Afrique de l'Est, de Somalie (Lanza, 1966). De celles-ci, nous connaissons jusqu'à présent 5 formes en Yougoslavie:

Stenasellus gjorgjevići gjorgjevići Racovitza, dans les grottes Ravanička pećina et Ivanjička pećina en Serbie,

Stenasellus skopljensis skopljensis Karaman, dans la nappe phréatique de Skoplje,

1) Zoologijski zavod, Prirodoslovno-matematički fakultet Sveučilišta, Rooseveltov $\operatorname{trg} 6$, Zagreb I, Jugoslavija. 
Stenasellus skopljensis croaticus Karaman, dans la nappe phréatique de Zagreb,

Stenasellus skopljensis meridionalis Karaman, dans les sources près de Dojran et Radovište en Macédoine

Stenasellus hungaricus thermalis Meštrov, dans les sources chaudes à Podsused, près de Zagreb.

L'espèce St. skopljensis Karaman, comme on le voit, est représentée par le plus grand nombre de formes et la plus vaste aire de répartition, des contrées Sud-Est de la Macédoine (Strumica) jusqu'aux contrées NordOuest de la Croatie (Zagreb). Cette trouvaille à Banja Luka suggère la possibilité d'autres découvertes à l'intérieur des limites connues jusqu'à présent.

Le corps des exemplaires de Banja Luka est allongé, étroit, de la même largeur sur toute sa longeur, mais uniquement un peu rétréci vers les extrémités de la tête et du pléotelson. Le rapport de la longueur du corps à la largeur est de $6: 1$ (fig. 1). La couleur est de la blancheur de la porcelaine. Les exemplaires adultes ont des dimensions plus petites ( $\sigma$ jusqu'à $5,5 \mathrm{~mm}$ et + jusqu'à $6 \mathrm{~mm}$ ) que ceux de St. skopljensis skopljensis (ơ jusqu'à $7 \mathrm{~mm}$, j jusqu'à $8 \mathrm{~mm}$ ). Par leur taille ils se rapprochent ainsi des sous-espèces meridionalis $(5 \mathrm{~mm})$ et croaticus $(05 \mathrm{~mm})$.

Le nombre des articles du flagelle de l'antenne I est de 10 et le nombre des aesthétases de 9 au maximum; elles sont légèrement plus longues que les articles auxquels elles appartiennent. En moyenne les ơ ont un nombre plus grand d'aesthétases que les ․ L'antenne II, comme pour le type, n'atteint pas le milieu du corps; repliée vers l'arrière elle arrive à toucher le bord postérieur du troisième péréionite libre. Il n'y a dans le flagelle que 20 à 25 articles, au lieu de 34 pour le type.

Le dactylopodite des péréiopodes I porte du côté intérieur seulement 4 épines $\left(0^{+} 4\right.$, 우 2$)$ et non 10 comme le type, tandis que le propodite a sur le bord intérieur seulement 8 épines caractéristiquement dentelées (fig. 2 et 2 ') et la forme typique jusqu'à 12 . Les péréiopodes $2-7$ portent une épine sternale (fig. 3).

Les pléopodes I ơ ont une construction typique (fig. 4). Le sympodite du pléopode II $\sigma$ est pentagonal, allongé. L'article proximal de l'endopodite est court et incorporé à l'article distal, comme chez les autres formes de l'espèce, mais on distingue clairement un sillon entre les deux articles. La partie proximale est étroite et la partie distale très élargie, et comme un bonnet il s'entasse sur une partie proximale de l'article 2. L'article distal est volumineux et se termine en pointe (fig. 5). Chez le St. skopljensis meridionalis la partie distale est obtuse, ce qui permet de distinguer essentiellement ces deux formes. La papille génitale a la forme d'un gros tube rond, rétréci à sa base et muni d'une large ouverture terminale. Les bases des papilles sont 
insérées à la partie antérieure médiane du sternite près des coxopodites du septième péréiopode (fig. 9). Le deuxième pléopode + a la forme typique d'un triangle, le rapport de la longeur à la largeur est de 1,5 (fig. 10), tandis que pour la forme typique la longueur et la largeur sont égales. Les autres pléopodes (III - V) sont conformes par leur construction à la forme typique. Chez toutes les sous-espèces leur endopodite est divisé dans sa longeur en deux petites languettes foliacées, ce qui est caractéristique de cette espèce européenne. Contrairement aux St. skopljensis meridionalis et croaticus, la languette extérieure de l'endopodite est plus longue que l'intérieure (fig. 6, $7,8)$; par celà cette sous-espèce de Bosnie diffère visiblement des St. skopljensis meridionalis et croaticus. Le deuxième article de l'exopodite du pléopode IV et partiellement le premier article du même appendice sont ridés et dentelés sur le bord intérieur, tandis que l'exopodite du pléopode $\mathrm{V}$ est recouvert d'écailles semi-lunaires avec un bord finement pectiné sur toute la longueur du côté intérieur et au sommet. Les uropodes sont plus courts que le pléotelson (rapport 1:1,5) (fig. 1), tandis qu'ils sont plus longs dans la forme typique.

Il résulte de cette description que les exemplaires du genre Stenasellus de la source chaude de Bosnie appartiennent incontestablement à l'espèce skopljensis, ce qui ressort spécialement de la forme des pléopodes II $\sigma^{\hat{t}}$ et de la caractéristique de la construction de l'endopodite des pléopodes III -V, qui sont totalement divisés longitudinalement. En plus de ces signes sont présentes d'autres caractéristiques de l'espèce skopljensis, décrites par S. Karaman $(1936,1948)$. Mais d'après les différences décrites ci-dessus par rapport au type et aux sous-espèces (S. Karaman, 1954), d'après l'analyse d'un plus grand nombre d'exemplaires ot et $q$ et d'après les caractéristiques du milieu, les animaux de Bosnie doivent être classés dans une sous-espèce particulierè Stenasellus skopljensis thermalis n. subsp.

Les espèces et sous-espèces du genre Stenasellus sont des habitants de différentes eaux souterraines. Si nous ne tenons compte que de l'espèce $S t$. skopljensis, on voit que les sous-espèces ne sont pas seulement morphologiquement mais aussi écologiquement différemment caractérisées. Ainsi le $S t$. skopljensis skopljensis et le St. skopljensis croaticus sont les habitants typiques des nappes phréatiques (S. Karaman, 1948, 1954) tandis que le St. skopljensis meridionalis est plus lié aux eaux souterraines courantes et sort à la surface dans les sources froides (S. Karaman, 1954). Le St. skopljensis thermalis diffère beaucoup plus des autres de ce point de vue, car il peuple les eaux souterraines de température plus élevée $\left(24^{\circ} \mathrm{C}\right)$ que la température moyenne de la region. Dans les mêmes conditions écologiques vit également le Stenasellus hungaricus thermalis (Meštrov, 1960) des sources chaudes de Podsused près de Zagreb. Des populations abondantes y sont constamment présentes et la biologie en est étudiée depuis un certain temps par le deuxième 
auteur de cet article. Jusqu'à présent il a été recueilli là plusieurs centaines d'exemplaires mais dans les sources froides voisines et les nappes phréatiques nous n'en avons point trouvé jusqu'à présent. Ceci nous prouve d'une façon convaincante que les eaux souterraines à température élevée ne sont pas un milieu anormal pour ces animaux, mais qu'elles sont le biotope de prédilection de ces formes.

L'espèce unique de l'Afrique de l'Est (Somalie) Stenasellus pardii Lanza vit également dans les sources à température plus élevée $\left(29^{\circ} \mathrm{C}\right)$ que la température moyenne de la région $\left(26,4^{\circ} \mathrm{C}\right)$ (Lanza, 1966).

Toutes les espèces eurasiennes du genre Stenasellus connues jusqu'à présent, excepté les deux mentionnées auparavant $-S t$. hungaricus thermalis et St. skopljensis thermalis en Yougoslavie - peuplent les eaux souterraines froides de température constante.

La présence du genre Stenasellus en Afrique orientale et dans les sources modérément chaudes $\left(18^{\circ}-24^{\circ} \mathrm{C}\right)$ de Yougoslavie est à la base de l'opinion selon laquelle il s'agit d'un relicte thermophile (A. Vandel, 1964). Les eaux thermales de ce type, c'est-à-dire les eaux modérément chaudes, ainsi que les autres eaux souterraines, sont des biotopes-refuges dans lesquels se sont maintenus également quelques formes relictes, ce qui est confirmé une fois de plus par ces deux découvertes des populations les plus abondantes du genre Stenasellus en Yougoslavie.

Novembre 1967

\section{RÉSUMÉ}

Une nouvelle sous-espéce de Stenasellus skopljensis thermalis de Banja Luka (Bosnie, Yougoslavie) est décrite.

Du point du vue écologique les St. skopljensis thermalis diffèrent fortement des autres sous-espèces, car ils peuplent les eaux souterraines à température plus élevée $\left(24^{\circ} \mathrm{C}\right)$ que la temperature moyenne de la région. Dans les mêmes conditions écologiques, dans les sources chaudes de Podsused près de Zagreb sur le territoire de Yougoslavie, vit également une autre espèce constamment abondante, St. hungaricus thermalis Meštrov. Cela prouve que ces eaux souterraines à température élevée ne sont pas un lieu de séjour anormal, mais le milieu préféré de ces formes, et confirme une fois de plus que les eaux thermales de ce type sont des biotopesrefuges dans les quels certaines formes relictes se sont maintenues.

\section{SUMMARY}

The new subspecies Stenasellus skopljensis thermalis, from Banja Luka (Bosnie, Yugoslavia) is described.

From the ecological point of view this form differs from the others because it inhabits underground waters of elevated temperature $\left(24^{\circ} \mathrm{C}\right)$. Another constantly abundant species, St. hungaricus thermalis Meštrov, also occurs in Yugoslavia under the same ecological conditions, in the warm springs of Podsused near Zagreb. This indicates that these underground waters at elevated temperature are not accidental but prefered habitats for these forms, and confirms once again that thermal waters of this type are the biotopes-refuges in which certain relic forms are retained. 


\section{BIBLIOGRAPHIE}

Karaman, S. (1936) - Ein Stenasellus aus dem Grundwasser von Skoplje, St. gjorgjevići skopljensis n. subsp. Glasnik skopskog naučnog društva, XVII, $137-141$.

- (1948) - O mužjacima izopoda Stenasellus skopljensis. Prirodoslovna istraživanja Jug. Akad. Zagreb, 24, 45-50.

- (1954) - Über die Jugoslavischen Stenasellus-Arten. Fragm. Balcanica Mus. Macedon. Sc. Nat., I, 2, 7-20.

LANZA, B. (1966) - Stenasellus pardii sp.n. della Somalia e note sistematiche su gli Stenasellinae (Crustacea, Isopoda). Monitore zoologico italiano, LXXIV, Suppl. $221-256$.

MAGNIEZ, G. (1966) - Contribution à la systematique des Stenasellinae (Crustacea) d'Afrique. International journal of speleology, II, 173-190.

Meštrov, M. (1960) - Stenasellus hungaricus thermalis ssp. n. (Crustacea, Isopoda), Nalaz predglacijalne vrste u toplim izvorima kod Zagreba, Biol. glasnik, $13,345-350$.

VANDEL, A. (1964) - Biospéologie - La biologie des animaux cavernicoles. Gauthier-Villars. Paris, $1-619$.

\section{EXPLICATION DES PLANCHES 97 (1) - 99 (3)}

Fig. 1. $\sigma^{7,7} \mathrm{~mm}$, le corps en vue dorsale. Fig. 2. ${ }^{7} 4,7 \mathrm{~mm}$, péréiopode 1 droit. Fig. 2'. $4,7 \mathrm{~mm}$, les phanères proximaux sur le bord sternal du propodite du péréiopode 1 droit. Fig. 3. $4,7 \mathrm{~mm}$, péréiopode 7 droit. Fig. $4.0^{7} 4 \mathrm{~mm}$, pléopodes 1, face sternale. Fig. 5. $\delta^{7}, 7 \mathrm{~mm}$, pléopodes 2, face sternale. Fig. $6 . \delta^{7} 4,7 \mathrm{~mm}$, pléopode 3 droit, face dorsale. Fig. 7. $\sigma^{7,7} \mathrm{~mm}$, pléopode 4 droit, face dorsale. Fig. 8. $\sigma^{\top} 4,7 \mathrm{~mm}$, pléopode 5 droit, face dorsale. Fig. 9 . $5 \mathrm{~mm}$, papille génitale en vue dorsale. a) basipodite du péréiopode 7 ; b) papille; c) tergite; d) coxopodite du péréiopode 7. Fig. 10. ㅇ $5 \mathrm{~mm}$, pléopodes 2, face sternale. 


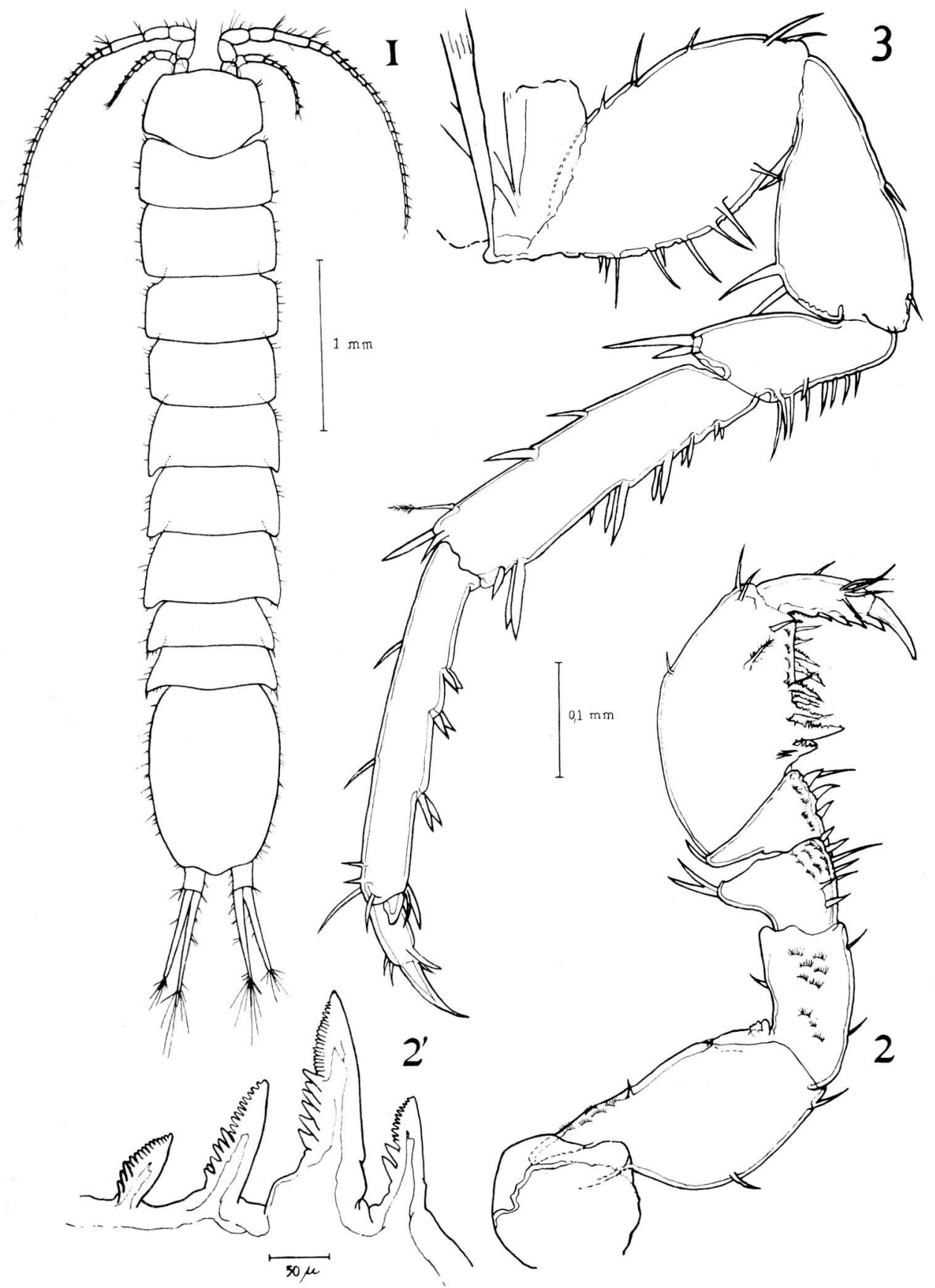



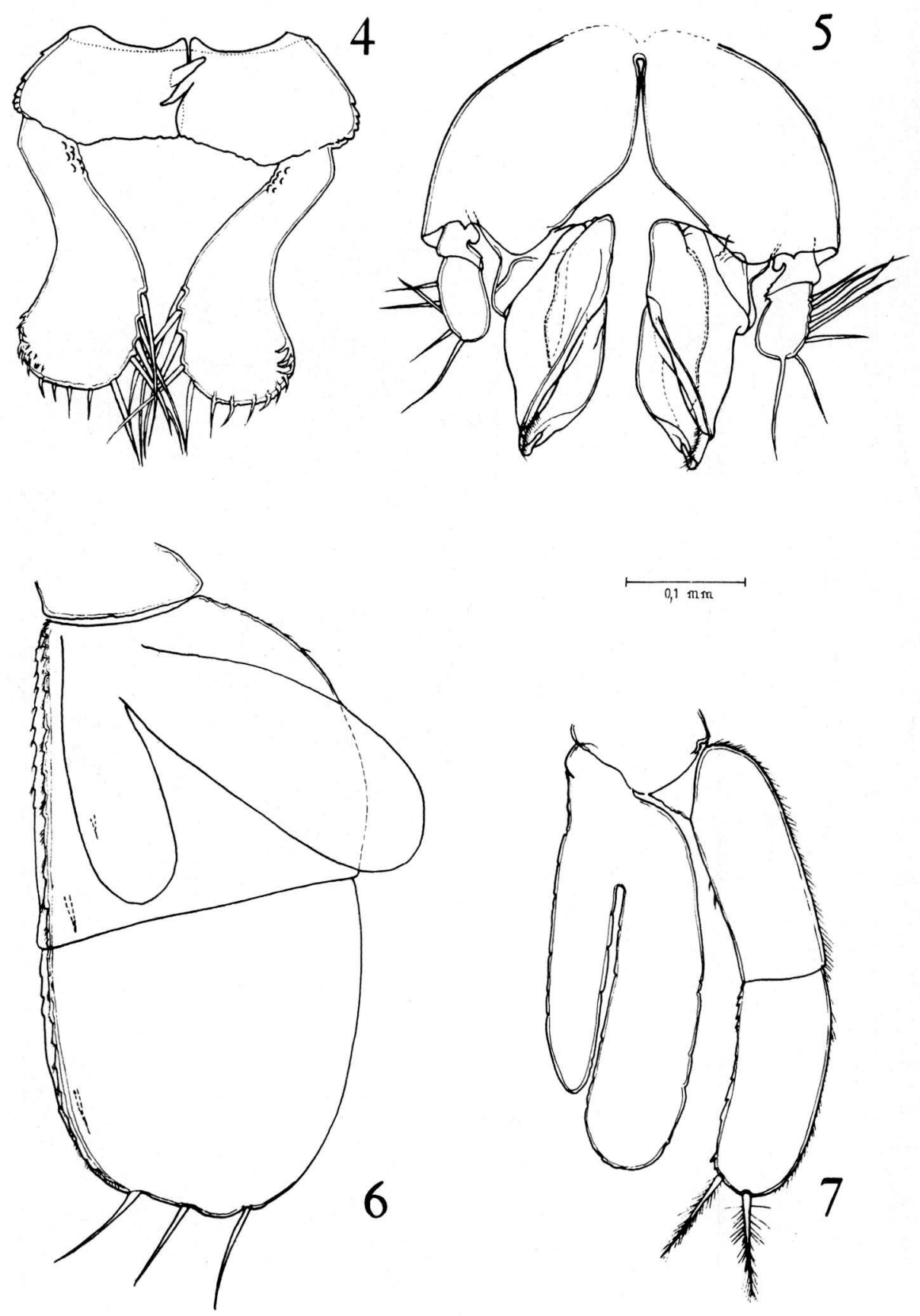

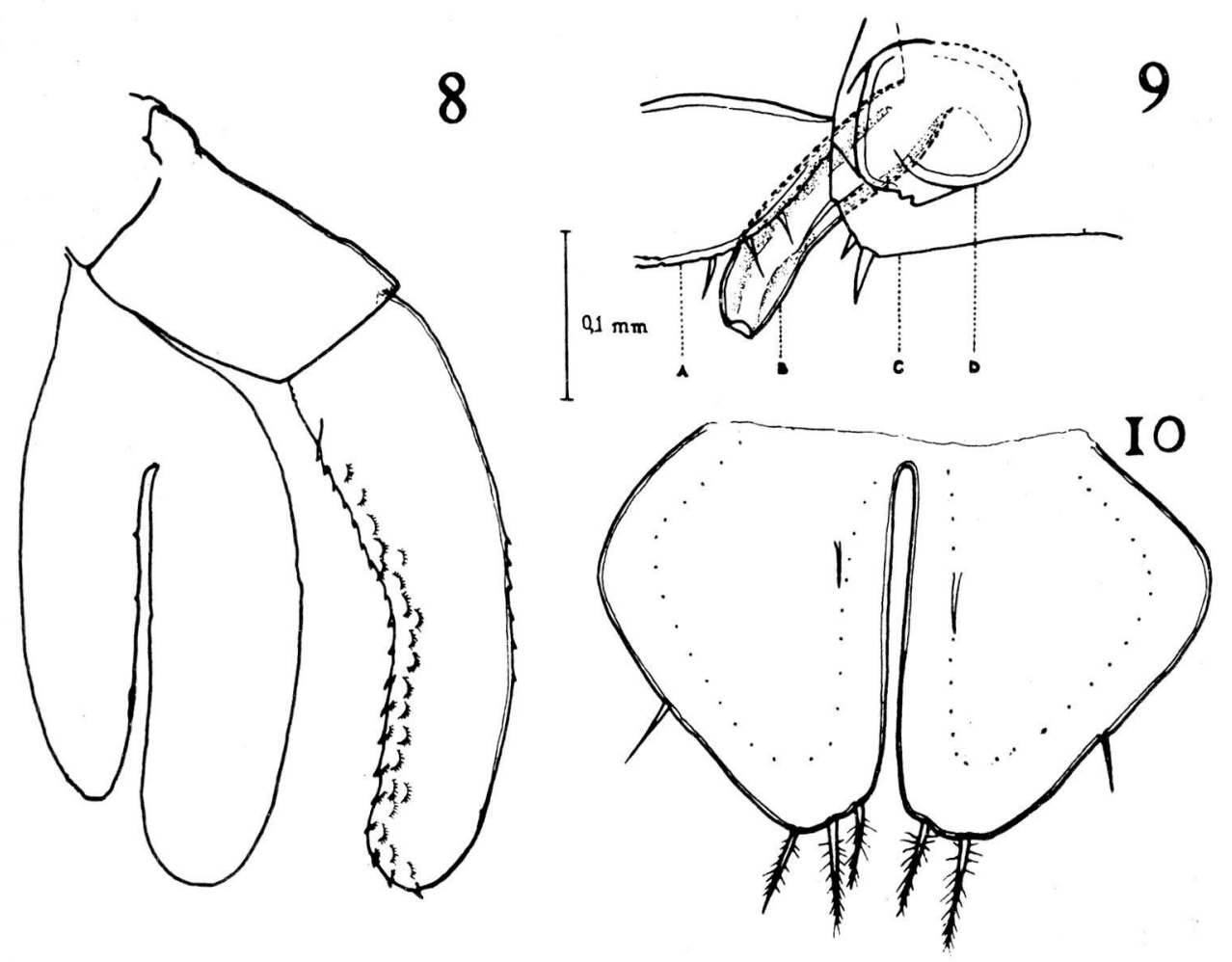